\title{
Il Giardeno, poema di imitazione dantesca del '400: edizioni promesse e citazioni reticenti in un secolo di bibliografia
}

\author{
Nicola De Blasi
}

È possibile che su un autore, per quanto di levatura non eccelsa, si susseguano durante un intero secolo titoli bibliografici ben poco informativi, se non completamente ripetitivi? Si direbbe di sì a giudicare dalle vicende toccate all'opera di Marino Jonata, poeta meridionale del Quattrocento, il cui caso può diventare forse esemplare per la conoscenza di una certa storia minore delle discipline filologiche, tanto da indurre a pensare che alla fortuna di Dante nel secolo $\mathrm{XV}$ corrisponda inesorabilmente la sfortuna bibliografica toccata nel corso del Novecento al suo tardo imitatore Marino Jonata.

1. Già nel 1851 il visconte Colomb de Batines, con un articolo sulla Etruria, ${ }^{1}$ annoverava tra le imitazioni dantesche del Quattrocento il Giardeno dello Jonata, stampato a Napoli nel 1490, e già registrato dai bibliografi, ma, precisava il De Batines, "pare che niuno di essi l'abbia, mai, veduto" (Imbriani 5). A questa segnalazione seguirono ulteriori informazioni e una prima parziale edizione, destinata a non restare l'unico tentativo di pubblicazione del Giardeno, mentre con una nota apparsa sul Giornale Storico della Letteratura Italiana, nel 1884 , veniva data notizia della scoperta di un manoscritto del poema (Nazionale di Napoli XIII C 13), ritrovato da Pasquale Papa, ${ }^{2}$ che era stato allievo di Vittorio Imbriani.

Questo annuncio suscitò una vivace reazione proprio da parte dell'Imbriani, pronto a ironizzare sul senso di una scoperta, a suo dire, insignificante o addirittura ridicola:

Può, mai, parlarsi, di scoperta d'un manoscritto, catalogato, in una biblioteca pubblica, sotto la stessa rubrica: ANGIONESE, sotto cui, è catalogata l'opera stampata? Scoperta? Sia pure! Però, in un senso relativo e subjettivo. Scoperta, solo, per riguardo, alla persona, che, prima, ne ignorava 
l'esistenza. Ma i compilatori ed i compulsatori precedenti, tutti, del catalogo, d'esso manoscritto conoscevano l'esistenza. ${ }^{3}$ (Imbriani 11)

Con il suo intervento infervorato, Imbriani sosteneva le ragioni di un giovane studioso, Francesco Ettari, che aveva diligentemente accolto il suo consiglio di dedicare la propria tesi di laurea al Giardeno di Jonata, a partire della lettura dell'incunabolo (Imbriani 8-10), ${ }^{4}$ e, in seguito, del manoscritto.

La tesi di laurea che coronò lo studio di Ettari apparve direttamente a stampa nel $1885:^{5}$ comprendeva una premessa su fonti e bibliografia, biografia dell'autore (tratta per lo più dalle note marginali del manoscritto), osservazioni sul poema (compresi alcuni ingenui confronti qualitativi con la Commedia!) e, merito più importante, la trascrizione, peraltro non priva di pecche, dei primi sette canti dell'opera, che nelle sue tre parti ne conta in tutto ben centosei. L'Ettari prometteva la pubblicazione completa del poema: ne fu invece distolto o dalla concorrente identica iniziativa ventilata da Pasquale Papa $^{6}$ o dalla dura recensione al suo lavoro. ${ }^{7}$

Il primo saggio di edizione (o, se si preferisce, di trascrizione) non è stato mai superato in seguito, né dall'Ettari stesso, né da altri: Pasquale Papa, infatti, si limitò a dare alle stampe un opuscolo, Per finirla, ${ }^{8}$ in cui riprendeva anche la polemica sul Giardeno in un contesto dai toni fortemente anti-imbrianeschi. Delle due annunciate edizioni, quindi, evento non rarissimo nella storia degli studi filologici, non se ne ebbe nessuna. O, almeno, non se ne ebbe nessuna completa, mentre furono pubblicate altre due edizioni parziali. La prima ad opera di Carlo Del Balzo, che incluse nella sua opera antologica sulla poesia di imitazione dantesca l'intero canto XI del Giardeno. ${ }^{9}$ La seconda fu invece procurata da Benedetto Croce ${ }^{10}$ con la pubblicazione di un brano del poema (di 45 versi) in lode della città dell'Aquila presente solo nel manoscritto (ff. 86-87) ed espunto dalla stampa del 1490.

Nel breve arco del biennio 1884-85 furono perciò prodotti gli scritti più significativi sul Giardeno. In seguito, se si escludono gli interventi di Del Balzo e di Croce, non appare più nulla di nuovo, a dispetto, ed è qui il dato da sottolineare, di un rincorrersi di contributi diversi.

2. Singolare è senza dubbio la sorte del lavoro editoriale di Ettari che, dimenticato o quasi in Italia, riapparve imprevedibilmente in 
una nuova veste linguistica, come risorto dall'oblio e come nuova pubblicazione, nella rivista americana Romanic Review, a quasi quarant'anni dalla prima apparizione napoletana e dalla recensione sul Giornale Storico. La redazione in lingua inglese è, rispetto a quella originale, un po' alleggerita nella parte introduttiva, e lievemente migliorate sembrano le cure dedicate alla trascrizione dei primi sette canti che restano i soli editi.

Potrebbe dirsi tardiva la decisione di ripubblicare, per quanto in altra lingua e in sede decisamente di maggior prestigio, un lavoro giovanile, ma forse l'Ettari, che tra l'altro non menziona la sua precedente pubblicazione, riteneva ormai sopite e dimenticate le polemiche che avevano accompagnato la sua tesi di laurea e constatava a giusta ragione che nessuna delle edizioni promesse era giunta a buon fine. Il numero della rivista americana con lo studio sul Giardeno fu segnalato, secondo la prassi consueta, dal Giornale Storico, ma nessun collegamento fu stabilito tra l'articolo americano dell'Ettari e la recensione di tanti anni prima al suo opuscolo napoletano. ${ }^{11}$

Già di per sé insolita fino a questo punto, la storia editoriale del Giardeno e della tesi di Ettari assume toni quasi inquietanti o addirittura perturbanti quando, dopo un altro intervallo di tempo di circa quarant'anni, nel 1966, l'estratto di Romanic Review è edito in un volume a sé che, uscito verosimilmente postumo, si configura come terza puntata di una storia editoriale distribuitasi lungo un ottantennio, passata attraverso le prime due fasi del 1885 e del 1924 (questa è la data del Copyright, detenuto dalla Columbia University Press di New York, dichiarata nel reprint) e caratterizzata, nonostante le due traduzioni e ristampe americane, da poca fortuna, almeno in termini di citazioni bibliografiche, come si può dedurre dagli accenni che altri hanno riservato al poema di Jonata.

3. Quasi mai al lavoro di Ettari è riconosciuto il riguardo che gli si dovrebbe, se non altro in termini di completa e corretta citazione, tanto che dai rimandi fatti da altri non è per niente chiaro che lo studioso napoletano abbia prodotto un'edizione parziale del testo, la più ampia mai pubblicata.

Non rende completa giustizia ad Ettari neanche Vittorio Rossi (ed è quanto dire!) che, mentre segnala con la consueta precisione, nel suo Quattrocento, il nesso tra la pubblicazione del 1885 e quella del 1923, offre solo un rinvio incompleto all'articolo americano: 
F. Ettari, El Giardeno di M. Jonata Agnonese poema del sec. XV, in Giorn. napol. di filosofia e lettere, 9, 1885, p. 772 sgg., e di nuovo, a b b r e v i a $t o$, in Romanic Review, 14, 1923, pp. 131-167 . . . (spaziatura aggiunta). (Rossi 278)

Il testo dei primi libri del Giardeno si trova stampato infatti alle pp. 131-167 del secondo fascicolo del 1923 della rivista, ma alle pp. 1-46 del primo fascicolo dello stesso anno si incontra la parte introduttiva del lavoro, che sfugge alla citazione del Rossi: la redazione americana risulta pertanto "abbreviata" rispetto alla napoletana. Del tutto irrilevante, come si può immaginare, tale svista del Rossi, mentre è invece notevole che dalla nota non risulti il tipo di contributo (edizione parziale o altro) offerto dall'Ettari.

La breve rassegna bibliografica dell'autore del volume di storia letteraria dedicato a tutto il Quattrocento italiano sarà stata presente all'Altamura che nel 1941 pubblicò un lavoro incentrato esclusivamente sul solo Umanesimo meridionale, dedicando perfino a Jonata un paragrafo corredato dai seguenti rinvii:

Cfr., precedentemente all'art. dell'Imbriani, la nota pubblicata dal Colomb De Batines nell'Etruria di Firenze (I, 1851, p. 391), che studiò il Giardeno nei tre esemplari della Palatina di Firenze, della Corsiniana e della Nazionale di Napoli. Per altre notizie v. F. Ettari, "El Giardeno" di M. Jonata Agnonese, (in Giorn. napol. di filos. e lett., IX, 1885, pp. 772 sgg., e di nuovo a b b revi a to, in Romanic Review, XIV, 1923, pp. 131-67)..." (spaziatura aggiunta). (Altamura, L'Umanesimo 74) ${ }^{12}$

Lo studio monografico (che avrà senz'altro avuto i suoi difetti) sembra quindi, secondo questo rinvio, contenere null'altro che notizie varie, e non una parte del testo del Giardeno (addirittura più particolareggiato, non si sa poi perché, è il rinvio al contributo del Colomb de Batines); e risalta ancor di più in questo caso anche l'assenza di ogni riferimento al Quattrocento di Rossi, che sin dalla prima edizione conteneva un accenno a Jonata (compreso quell'abbreviato che, inserito nella terza edizione, diventa quasi un errore-guida in questa piccola ricostruzione bibliografica), laddove nel corso del nostro secolo la tendenza è stata quella di far passare l'Agnonese per un personaggio del tutto ignoto agli italianisti.

Da una rassegna bibliografica concepita come quella appena letta fino alla scomparsa completa del nome di Ettari il passo è breve, e si realizza in pieno finanche in studi di altissimo livello sul Quattro- 
cento napoletano che però sfiorano appena il Giardeno. Ciò accade nella fondamentale edizione delle Rime di De Jennaro procurata da M. Corti, che nel citare Jonata (il cui poema può essere in verità a stento accostato alle Sei etate di De Jennaro) dà le seguenti notizie sui titoli precedenti:

Cfr. il Giardeno di M. Jonata da Agnone (Imbriani, Notizie di M. Jonata Agnonese, in Rend. R. Acc. di sc. mor. e pol. di Napoli, XXIV (1885), pp. 44-77; Altamura, Uman., pp. 73-74 con bibliog.) in tre cantiche, terminato nel 1465, (Croce, Ricerche di antica letteratura meridionale, Arch. stor. nap. LVI (1931), pp. 5-86, a p. 44). (De Jennaro xv)

Solo per completare le informazioni di cornice intorno all'opera di De Jennaro la Corti inserisce un riferimento, necessariamente fugace, al Giardeno di Jonata e si limita ad un veloce rinvio ad una bibliografia che solo ad un primo sguardo, come si è visto, può apparire completa. Non rimangono tracce del nome di Francesco Ettari, né del suo lavoro, neanche nel capitolo sulla letteratura volgare in età aragonese della collettiva Storia di Napoli; in questa occasione, infatti, A. Altamura, autore del contributo, nel citare alcuni brani del poema di Jonata, prescinde da ogni completo riferimento bibliografico e rinvia soltanto a sé stesso. ${ }^{13}$

Neanche nell'opera che costituisce in genere la prima e più immediata fonte di informazione, se non il punto di partenza, per chi si occupi di storia culturale napoletana e di antichi testi del Regno di Napoli, vi è quindi notizia di una parziale edizione del Giardeno, né può sorgere alcun sospetto in merito poiché il testo è presentato come "un altro poemetto pressoché sconosciuto" (Altamura, "Napoli aragonese" 503) di modo che dell'esistenza del lavoro di Ettari si può venire al corrente solo per via di indiscrezioni o grazie al rinvenimento casuale di articoli ottocenteschi o di estratti recenti sempre meno citati.

Pressoché "sconosciuto" il poema è ancora nel 1976, stando alle informazioni che l'Altamura raccoglie sugli imitatori meridionali di Dante, da tempo in verità non più ignoti per merito di lavori dello stesso o di altri studiosi. ${ }^{14}$ Dieci anni dopo l'edizione americana in estratto del saggio di Ettari, non ve ne è notizia nella nota iniziale del volume sugli imitatori danteschi, che cita solo il primo lavoro di Ettari e si limita a ricordare gli scritti di Albino, Imbriani, Ciampa (un magistrato di Agnone, già contraddetto cinquantacinque anni prima 
da Croce), Del Balzo, Casa, Croce, nonché dello stesso Altamura. Il nome di Ettari in verità ricorre per primo ma, anche qui, in un contesto piuttosto sibillino:

Per notizie sul Gionata cfr. F. Ettari nel "Giornale napoletano di filosofia e lettere," IX (1885), fascicoli 32-33. (Altamura-Basile 57)

Manca in tal modo la possibilità di sapere quale differenza possa sussistere tra il lavoro di Ettari e gli altri citati. Ne consegue, pertanto, che qualsiasi lettore, in assenza di informazioni precise, è indotto a credere che brani del Giardeno siano pubblicati per la prima volta proprio dall'Altamura, che nel corso del capitolo dedicato al poema (cui, in verità, mal si addice, date le dimensioni, il ricorrente appellativo di poemetto), ne trascrive a più riprese dei passi, per un totale di centosettantotto versi, tutti dai primi sette libri del Giardeno, gli stessi pubblicati da Ettari. Occorre tuttavia segnalare la maggior perizia di Altamura nella trascrizione che presenta separazione di parole e punteggiatura, mentre Ettari aveva trascritto pressoché diplomaticamente. ${ }^{15}$

Ancora nel 1978 altri passi del Giardeno (sempre dai primi sette libri) sono antologizzati da Altamura (La lirica 53-58), ma la situazione non cambia rispetto alle informazioni bibliografiche date in precedenza, che qui ritornano anzi in una nota redatta negli stessi precisi termini di quella del volume sulle Imitazioni.

4. Dalle tre cadenzate edizioni parziali dell'Ettari, dalle segnalazioni successive dell'Altamura, dalle promesse edizioni di Papa e Albino, dalle notizie di volta in volta apparse sul Giornale storico, si direbbe, in conclusione, che il Giardeno di Marino Jonata abbia finora conosciuto solo studiosi disposti a dedicargli le attenzioni sufficienti per un primo esame, una prima valutazione o, nella migliore delle ipotesi, un iniziale saggio di edizione con promessa di integrazione successiva.

Novità assoluta ormai il Giardeno non è più da tempo e non può essere più (si spera) segnalato come tale, mentre resta ancora in attesa di uno studio complessivo che ne valuti anche le interessanti caratteristiche linguistiche. Questa breve nota ha inteso soltanto proporre lo strano caso di un testo fin troppo segnalato, più volte parzialmente edito, ma in sostanza ancora da studiare, nonostante una bibliografia non poverissima, e si è limitata a prospettare la necessità, per chi voglia studiare il Giardeno, di risalire agli scritti di Ettari e di Im- 
briani (o, meglio ancora, a Jonata direttamente), prescindendo (con l'eccezione delle pagine di Croce) dalla pur vasta serie di apparenti contributi novecenteschi sull'argomento.

\section{Università della Basilicata}

\section{NOTE}

1 L'articolo, "Domenico de Napoli e Marino Yonata Angionese poco conosciuti del secolo xv," è riportato interamente da Vittorio Imbriani, che scrive così: "Ecco lo articoletto del bibliofilo e dantofilo oltramontano, che si mostrava più zelante ed operoso de' letterati Italiani"(4). Angionese è da intendere come agnonese, cioè originario di Agnone, oggi nel Molise. Il poema è tra le primissime attestazioni letterarie del volgare di età aragonese a Napoli (precede di qualche anno la produzione dei lirici di corte): la lingua è meridionale, con tratti caratteristici di area abruzzese.

2 "Il signor Pasquale Papa ha rinvenuto nella Biblioteca Nazionale di Napoli un ms. del Giardeno poema in terza rima del quattrocentista Marino Jonata di Agerone (sic), condotto ad imitazione della Divina Commedia. È il primo ms. che di questo poema giunge a notizia degli studiosi, e la sua importanza è accresciuta dall'esservi il poema corredato di un commento dovuto allo stesso autore, che offre qualche lume di storiche notizie intorno alla sua vita, di cui fin qui non si sapeva che pochissimo. Il poema sarà pubblicato a cura del sig. Papa e del sig. Francesco Ettari che già attende a trascriverlo" (Giornale storico della Lelteratura Italiana 3 [1884]: 476). Il commento marginale che correda il testo del ms. è in latino. Un annuncio uscì anche sul Pungolo e sul Fanfulla della Domenica (P. Papa, Per finirla 4).

3 Ben più espressiva la chiusa dell'ironico capoverso sulla scoperta del Papa: "Cosi, chi vedesse scollacciata, per la prima volta, una bella donna, potrebbero (sic) scoprirle un neo, sull'omero, già, similmente, scoperto, da quanti l'han visto, prima. E chiunque capita, a Napoli, può scoprire Fontana Medina e la Riviera di Chiaja!" (Imbriani 11).

4 F. Ettari è lodato per aver presentato già stampata la sua tesi di laurea alla Facoltà, per aver accettato il consiglio di Imbriani: "A più d'uno, suggerii di scêrre il Giardeno, per tema d'una monografia o della tesi di laurea. Parecchi s'acceser di zelo . . . o fecer le viste; e, poi, indietreggiarono. Non così il professor Francesco Ettari. .. ," (Imbriani 8), e per aver lavorato durante un intero anno ("l'anno scorso," quindi nel 1884) mentre imperversava in Napoli un'epidemia di colera che provocò la morte di un fratello dello stesso Ettari: "che, ingenuamente caritatevole, accudiva i colerosi, ascrittosi, ad una delle brigate, che gente furba organizzò ... perché? . . Vattelappesca!" (Imbriani 8). 
5 El Giardeno di Marino Jonata Agnonese poema del secolo XV, tesi di laurea di Ettari Francesco, Estratto dal Giornale Napoletano di filosofia e Lettere 9:3233 (1885):1-72. La copia di questa pubblicazione conservata alla Nazionale di Napoli porta la firma di Ettari Francesco e la data, 7 Gennaio 1885.

6 “'L'Ettari promette di continuare le sue indagini, intorno a Marino Jonata; (.. .) Ov'egli si disanimasse o indugiasse, troppo, possiamo, credo, fare assegnamento, sul Papa, giovane serio e solerte e sollecito, che (obbligato, dalla sua parola!) farà, di tutto, per ripubblicare il Giardeno: od accordandosi o gareggiando, con l'Ettari. Sarebbe, davvero, peccato, che il materiale, ch'esso Papa deve avere, in pronto, e gli studi, ch'e' deve aver fatti (secondo l'annunzio, che, come ho detto, fece inserire, ne' giornali!) andassero perduti. E (chi sa? chi sa?) dopo tanto oblio, forse, a breve intervallo, ci avremo due ristampe e due illustratori del poema dello Angionese. Ex privatis odiis respublica crescit." (Imbriani 27). La scoperta del Papa, come si è già segnalato, fu annunciata anche dal Fanfulla della Domenica e dal Pungolo (Papa, Per finirla 4).

7 Piuttosto severo il giudizio sulle capacità filologiche dell'Ettari, che avrebbe trascritto il testo "senza correggere gli errori manifesti, senza cercare di far tornare i versi (. . .) senza aggiungere di suo neppure la indispensabile punteggiatura. Una buona metà di questo opuscolo è opera d'amanuense" ("Recensione" 456); ancor più netto il parere del recensore a proposito di una completa pubblicazione del poema: "Quanto sia opportuna tale pubblicazione non sappiamo dire: quel che ci sembra certo è che il sig. Ettari non è in alcun modo preparato ad un lavoro simile. Una pura ristampa del poema, nel modo come furono dati i sette canti di saggio, sarebbe una sconcezza" ("Recensione" 457). La recensione, non firmata, può forse attribuirsi a Rodolfo Renier, se può valere come indizio il fatto che egli in seguito si sarebbe in due occasioni interessato alle opere del De Jennaro, poeta della corte aragonese, contemporaneo, o quasi, di Jonata. (Renier, "Notizia" 248 [si tratta delle Sei etate di De Jennaro] e "Opere inesplorate"). In questa recensione sembra di poter intravedere non tanto un diretto attacco al giovane Ettari, quanto un conflitto tra due diversi modi di concepire il contatto con gli antichi testi. Un giudizio limitativo è infatti espresso anche sulla relazione dell'Imbriani, che, a dire dell'estensore della nota di segnalazione, "tratta del Giardeno, con maggior copia d'erudizione e con maggior oculatezza (se non con metodo molto migliore) di quello che abbia fatto il sig. F. Ettari." ("Notizia redazionale," Giornale storico della Letteratura Italiana 6:309).

8 La storia del ritrovamento del ms. ("cercatolo a bella posta"), dell'incontro tra Papa ed Ettari in Biblioteca, della generosità del Papa stesso ("si fissò di fare assieme il lavoro") è narrata alle pp. 3-7, inserita all'interno di una tirata polemica contro Gaetano Amalfi, che aveva accusato A. Bartoli di aver plagiato Imbriani. L'episodio del Giardeno è narrato a dimostrazione della "buona fede della ditta" Amalfi-Imbriani, ovvero del "suggeritore" e del "procuratore" dell'Ettari (4). Altri interventi nella polemica sul presunto plagio di Bartoli: 
P. Papa, "Ed ora il signor Gaetano Amalfi con un libello meditato un annetto viene a dirci che il volume della Storia della Letteratura Italiana del Bartoli è un plagio da cima a fondo" (Sul quinto volume) c G. Amalfi: "Il Bartoli segue, passo passo, l'Imbriani ..." (Adolfica). "Imbriante" è considerata da Papa la tendenza di Amalfi a vedere Imbriani dappertutto.

9 C. Del Balzo, Poesie 73-83. Del Balzo riproduce il testo dall'incunabolo e nel rammentare la bibliografia precedente segnala che Imbriani è intervenuto "aggiungendo poche cose alle già dette dall'Ettari, rinverdendo polemiche e pettegolezzi tra il Papa e l'Ettari." Inoltre informa che l'avv. Pasquale Albino di Campobasso, direttore della Biblioteca Molisana, collezione editoriale delle opere scritte da autori molisani, aveva iniziato la riproduzione dell'incunabolo presso la tipografia di E. Perino editore di Roma. L'Albino, autore di un articolo apparso su "La Nuova Provincia del Molise" nel 1885, verosimilmente non ha portato a termine il suo progetto, che annunciò forse solo al fine di entrare in lizza nella polemica tra Papa ed Ettari, cercando di superare entrambi nella gara degli annunci.

10 B. Croce, "Poesia volgare" ora in Aneddoti 53-55.

11 A proposito del primo fascicolo della rivista, della prima metà del lavoro di Ettari, si legge: "L'A. dà notizia del ms. di questo poemetto del XV sec., dell'incunabolo, tratta la biografia dell'autore e mette in risalto le caratteristiche del componimento" (Segnalazione di Ettari, Giornale Storico, 412). La continuazione del contributo sul fascicolo 2-3 è così commentata: "L'A. pubblica il testo di questo poemetto." In questa nota informativa sul sommario di Romanic Review il Giardeno è per la prima volta definito "poemetto" forse perché lo si ritiene pubblicato per intero nelle circa trenta pagine della seconda parte dell'articolo di Ettari.

12 Altri contributi citati sono quelli di F. Casa e V. Zabughin (pp. 124-28 e 16869) e gli scritti di N. Ciampa citati da Croce. In un lavoro di "bibliografie e testi inediti" è proprio la bibliografia a mostrare dei lati deboli.

13 A. Altamura, "Napoli aragonese. La letteratura" 565, nota 5, che si riferisce al Rosarium de spinis di fra' Domenico Mercari dell'ordine del Predicatori, detto Domenico da Napoli: "Ne diedi notizia per primo nel 'Giornale Dantesco,' XLII (1941) pp. 93 sgg. $-\mathrm{Nel}$ medesimo scritto si trovano notizie anche sui consimili poemetti del Gionata e del De lennaro." Il fra Domenico Mercari è lo stesso la cui opera (stampata nel 1475) è segnalata da Colomb de Batines, "Domenico de Napoli" 5 dell'art. cit. di V. Imbriani, contributi che altrove risultano entrambi noti all'Altamura (cfr. L'Umanesimo).

14 A. Altamura-P. Basile: il capitolo su Jonata (che anche qui diventa Gionata) è a pp. 57-68, ma non è chiaro cosa di questo capitolo sia dovuto ad A. Altamura e cosa a Pina Basile, poiché non esiste nessun indizio, né alcuna nota dichiarativa, in tal senso. Il Giardeno, ad ogni buon conto, è ancora presentato come "pressoché sconosciuto" (p. 57).

15 Non sono invece illuminanti le notizie biografiche riportate nel volume: a p. 58 si dice che Jonata sarebbe morto "dopo il ' 75 , ma non prima del ' 90 
(allorché il figlio pubblicò, postumo, il poemetto paterno)," dove l'unico dato certo il terminus ad quem del 1490 diventa, forse anche per un perfido errore di stampa, un secondo terminus a quo, per cui viene involontariamente affermato che la data di morte di Jonata è sì dopo il 1475, ma è dopo ("non prima del") il 1490.

\section{OPERE CITATE}

Altamura, Antonio. La lirica napoletana del Quattrocento. Napoli: Società Editrice Napoletana, 1978.

. L'Umanesimo nel Mezzogiorno d'Italia. Storia, bibliografie e testi inediti. Firenze: Bibliopolis, 1941.

"Napoli aragonese. La letteratura." Storia di Napoli. Vol. 4. Tomo 2. Napoli: ESI, 1974. 501-71.

Altamura, Antonio e Pina Basile. Imitazioni dantesche di quattrocentisti meridionali. Napoli: Società Editrice Napoletana, 1976.

Amalfi, Gaetano. Adolfica (Se vi sia reato di plagio nel quinto volume della Storia della letteratura italiana di A. Bartoli). Napoli, 1884.

Casa, F. "Un poema del sec. XV." In Studi e rassegne. Città di Castello, 1899.

Croce, Benedetto. "Poesia volgare a Napoli nella prima metà del Quattrocento." Archivio storico per le provincie napoletane 46 (1931): 5-86. Ora in Aneddoti di varia letteratura. $2^{\text {a }}$ ed. Vol. 1. Bari: Laterza, 1953. 33-58.

De Batines, Colomb. "Domenico de Napoli e Marino Yonata Angionese poco conosciuti del secolo XV." Etruria 1 (1851): 391 sgg.

De Jennaro, Pietro Jacopo. Rime e lettere. A cura di Maria Corti. Bologna: Commissione per i testi di lingua, 1956.

Del Balzo, Carlo. Poesie di mille autori intorno a Dante Alighieri. Vol. 4. Roma, 1893.

Ettari, Francesco. "El Giardeno di Marino Jonata Agnonese poema del secolo XV." Tesi di laurea. Giornale napoletano di filosofia e lettere 9. 32-33 (1885): $1-72$.

Ettari, Francesco. "El Giardeno of Marino Jonata Agnonese: an Italian Poem of the Fifteenth Century." Romanic Review 14. 1 (1923): 1-46 e Romanic Review 14. 2-3 (1923): 131-67.

Imbriani, Vittorio. "Notizie di Marino Jonata Agnonese." Rendiconto dell'Accademia di Scienze morali e politiche di Napoli 24 (1885): 4-7.

"Notizia redazionale." Giornale storico della letteratura italiana 3 (1884): 476.

"Notizia redazionale." Giornale storico della letteratura italiana 6 (1885): 309.

Papa, Pasquale. Per finirla. Firenze, 1885.

Sul quinto volume della Storia della letteratura italiana del prof. A. Bartoli. Firenze, 1884.

"Recensione a Francesco Ettari. 'El Giardeno di Marino Jonata Agnonese poema del secolo XV.' Tesi di laurea. Giornale napoletano di filosofia e lettere 9. 32-33 (1885): 1-72." Giornale storico della letteratura italiana 5 (1885): 455-57. 
"Segnalazione di Ettari, Francesco. 'El Giardeno of Marino Jonata Agnonese: an Italian Poem of the Fifteenth Century.' Romanic Retiew 14. 1 (1923): 1-46 e Romanic Review 14. 2-3 (1923): 131-67." Giornale storico della letteratura italiana 86. 1 (1925): 412.

Renier, Rodolfo. "Notizia di un poema inedito napolitano." Giornale storico della letteratura italiana 8 (1886): 248-58.

"Opere inesplorate del Di Gennaro." Giornale storico della letteratura italiana 11 (1888): 469-75.

Rossi, Vittorio. Il Quattrocento. $3^{3}$ ed. Milano: Vallardi, 1938. Vol. 4 della Storia letteraria d'ltalia.

Zabughin, Vladimiro. L'oltretomba classico medievale nel Rinascimento. Firenze-Roma: Olschki, 1922. 